Published in final edited form as:

Oncogene. 2018 November ; 37(45): 5982-5996. doi:10.1038/s41388-018-0387-9.

\title{
ZFPM2-AS1, a Novel IncRNA, Attenuates the p53 Pathway and Promotes Gastric Carcinogenesis by Stabilizing MIF
}

\author{
Fanyang Kong ${ }^{1,2}$, Xuan Deng ${ }^{2,3}$, Xiangyu Kong ${ }^{1,2}$, Yiqi $\mathrm{Du}^{2}$, Lei $\mathbf{L i}^{2}$, Huiyun $\mathrm{Zhu}^{2}$, Yuxin \\ Wang $^{2}$, Dacheng Xie ${ }^{1}$, Shivani Guha ${ }^{1}$, Zhaoshen $\mathrm{Li}^{2}$, Ming Guan ${ }^{3}$, and Keping Xie ${ }^{1}$ \\ ${ }^{1}$ Department of Gastroenterology, Hepatology \& Nutrition, The University of Texas MD Anderson \\ Cancer Center, Houston, Texas \\ 2Department of Gastroenterology, Changhai Hospital, Second Military Medical University, \\ Shanghai, People's Republic of China \\ ${ }^{3}$ Department of Laboratory Medicine, Huashan Hospital, Shanghai Medical College, Fudan \\ University, Shanghai, People's Republic of China
}

\begin{abstract}
Background-Long non-coding RNAs (lncRNAs) are implicated to be involved in the pathogenesis of many cancers. Herein we report on our discovery of a novel lncRNA, ZFPM2 antisense RNA 1 (ZFPM2-AS1), and its critical role in gastric carcinogenesis.
\end{abstract}

Methods-ZFPM2-AS1 expression in gastric cancer specimens was analyzed using Gene Expression Omnibus data set and validated in 73 paired gastric tumor and normal adjacent gastric tissue specimens using qRT-PCR. The effect of ZFPM2-AS1 expression on proliferation and apoptosis in gastric cancer cells was assessed by altering its expression in vitro and in vivo. Mechanistic investigation was carried out using cell and molecular biological approaches.

Results-ZFPM2-AS1 expression was higher in gastric tumors than in normal gastric tissue. Also, increased ZFPM2-AS1 expression in gastric cancer specimens was associated with tumor size, depth of tumor invasion, differentiation grade, and TNM stage. High ZFPM2-AS1 expression predicted markedly reduced overall and disease-free survival in gastric cancer patients. Functional experiments demonstrated that ZFPM2-AS1 expression promoted proliferation and suppressed apoptosis of gastric cancer cells in vitro and promoted tumor growth in vivo. This effect is associated with attenuated nuclear translocation of p53. Mechanistic experiments demonstrated that tumor-activated ZFPM2-AS1 could bind to and protect the degradation of macrophage

\footnotetext{
Users may view, print, copy, and download text and data-mine the content in such documents, for the purposes of academic research, subject always to the full Conditions of use: http://www.nature.com/authors/editorial_policies/license.html\#terms

Corresponding Authors: Keping Xie, Department of Gastroenterology, Hepatology and Nutrition, Unit 1466, The University of Texas MD Anderson Cancer Center, 1515 Holcombe Boulevard, Houston, TX 77030. Phone: 1-713-794-5073; Fax: 1-713-745-3654; kepxie@mdanderson.org; Zhaoshen Li, Department of Gastroenterology, Changhai Hospital, Second Military Medical University, Shanghai, People's Republic of China. Phone: 86-21-31161344; zhaoshenli5610@ hotmail.com; and Ming Guan, Department of Laboratory Medicine, Huashan Hospital, Shanghai Medical College, Fudan University, Shanghai, People's Republic of China. Phone: +86021 52888048; guanminghsyy@ hotmail.com.

F. Kong, X. Deng, and X. Kong are co-first authors.

Disclosure of Potential Conflicts of Interest: The authors declare no conflicts of interest.
} 
migration inhibitory factor (MIF), a potent destabilizer of p53. Knockdown of MIF expression diminished ZFPM2-AS1's impact on p53 expression in gastric cancer cells.

Conclusions-Our findings demonstrated that ZFPM2-AS1 regulates gastric cancer progression and revealed a novel ZFPM2-AS1/MIF/p53 signaling axis, shedding light on the molecular mechanisms underlying the tumorigenicity of certain malignant gastric cells.

\section{Keywords}

LncRNA; MIF; P53; Gastric cancer; progression

\section{Introduction}

Gastric cancer is one of the most frequently diagnosed cancers and a leading cause of cancer-related deaths worldwide ${ }^{1,2}$. Strikingly, cases of gastric cancer in China alone account for $42 \%$ of all cases worldwide, probably owing to the high prevalence of Helicobacter pylori infection in the Chinese population ${ }^{3}$. Although researchers have made massive efforts to develop noninvasive biomarkers and therapeutic targets to reduce gastric cancer mortality 4,5 , they have had little success in increasing the overall survival rate. Therefore, discovery of new diagnostic and prognostic markers and an improved understanding of molecular mechanisms of gastric tumorigenesis remain urgent needs.

Long non-coding RNAs (lncRNAs), a class of noncoding transcripts, are longer than 200 nucleotides, with little protein-coding potential. LncRNAs are abundantly transcribed in developmental stage-, tissue-, or disease-specific manner ${ }^{6,7}$. Recently, accumulating evidence has demonstrated that IncRNAs are critical regulators in a variety of cellular processes via control of expression of multiple genes involved in the development and progression of various tumors, including gastric cancer ${ }^{8,9}$. LncRNA expression profiling may facilitate the diagnosis of and prognosis for gastric cancer, which may serve as effective therapeutic targets for gastric cancer intervention. However, although alteration of lncRNAs in gastric tumors is a widely recognized, roles of many gastric cancer-associated lncRNAs and the related molecular mechanisms remain largely undetermined.

Antisense lncRNAs are RNAs that are reverse complements of their endogenous sense counterparts ${ }^{10}$. Antisense transcripts comprise a big proportion of long on0coding transcriptome (50-70\%) ${ }^{11,12}$. Due to their high locus-specification, the biology importance of antisense transcripts was overlooked for decades. It is elucidated recently that antisense transcripts, like many characterized lncRNAs, exert trans and cis effects on other genes ${ }^{13}, 14$ and leading to gene promoter activation or posttranscriptional regulation by controlling mRNA and protein stability.

In the present study, we identified the lncRNA ZFPM2 antisense RNA 1 (ZFPM2-AS1) as a candidate oncogene involved in gastric cancer progression using information from the Gene Expression Omnibus and The Cancer Genome Atlas (TCGA) data sets. We then systematically verified and validated the functions of ZFPM2-AS1 using human specimens, cell and molecular biological studies, and animal models. We first determined the expression of ZFPM2-AS1 in gastric tumors and its correlation with clinical aggressiveness and poor 
survival. We then investigated the impacts of altered expression of ZFPM2-AS1 on gastric cancer cell proliferation, cell-cycle progression, and apoptosis. Inactivation of P53 is one of the most common event in gastric carcinogenesis. Recent studies have suggested MIF, a 12.5 $\mathrm{kDa}$ cytokine, may be involved in carcinogenesis through inactivation of $\mathrm{p} 53^{15}$, promotion of angiogenesis ${ }^{16}$, as well as a Rho dependent pathway ${ }^{17}$. Here, we determined that ZFPM2AS1 attenuated the p53 signaling pathway via physical interaction with and upregulation of expression of macrophage migration inhibitory factor (MIF) in gastric cancer cells.

\section{Results}

\section{Identification and characterization of ZFPM2-AS1 expression}

By analyzing data from the Gene Expression Omnibus data set, we found that ZFPM2-AS1, was expressed at higher levels in gastric cancer than in gastric tubular adenoma specimens (Supplementary Fig. S1A). To further clarify the role of expression of ZFPM2-AS1 in gastric cancer specimens, we extracted and examined 375 gastric cancer specimens and 32 normal gastric tissue specimens from TCGA data portal. ZFPM2-AS1 expression was markedly higher in gastric cancer than in normal tissue specimens (Supplementary Fig. S1B), and high ZFPM2-AS1 expression was associated with poor survival (Supplementary Fig. S1C). Furthermore, we analyzed ZFPM2-AS1 gene expression data on colorectal, liver, and esophageal cancer specimens and observed similar results (Supplementary Fig. S1D-F), suggesting that upregulation of ZFPM2-AS1 expression is common in tumor cells during cancer progression. Therefore, we assumed that that ZFPM2-AS1 plays a carcinogenic role regarding gastric cancer.

Unexpectedly, our 5' and 3' RACE assays identified a novel ZFPM2-AS1 transcript (1168 bp) containing three exons (E1, 59 nt; E2, 118 nt; and E3, 991 nt) (Fig. 1A and B). The fulllength ZFPM2-AS1 sequence is shown in Supplementary Fig. S2. We verified that ZFPM2AS1 is a non-coding RNA using three online protein-coding potential assessment software programs (Supplementary Fig. S3A-C). We treated AGS cells with the DNA methylation inhibitor 5-azacytidine but find no change of ZFPM2-AS1 expression in them (Supplementary Fig. S4A). Furthermore, we treated AGS cells with the histone deacetylase inhibitor trichostatin A and determined that expression of ZFPM2-AS1 was markedly upregulated in them (Supplementary Fig. S4B). These results indicated that ZFPM2-AS1 expression in gastric cancer cells may be regulated by histone acetylation.

\section{ZFPM2-AS1 expression is frequently upregulated in gastric cancer and closely correlated with prognosis}

To clarify the expression pattern for the novel transcript ZFPM2-AS1 in gastric tumors, we examined its expression using specific primers in 73 paired gastric tumor and normal adjacent gastric tissue specimens using qRT-PCR. The results demonstrated that expression of ZFPM2-AS1 was considerably higher in the cancer than in the normal tissue specimens (Fig. 1C and D). Also, the level of ZFPM2-AS1 expression in a nonmalignant gastric cell line was markedly lower than that in gastric cancer cell lines (Fig. 1E). Furthermore, we used the normal adjacent tissue specimens as controls to produce a receiver operating 
characteristic curve. The area under the receiver operating characteristic curve was 0.760 (95\% confidence interval, $0.647-0.846 ; P=0$ ) (Fig. 1F).

Based on the median ratio of ZFPM2-AS1 expression in tumor specimens, all the patients were classified into highor low ZFPM2-AS1 expression group. To determine the clinical value of ZFPM2-AS1 expression, we analyzed the correlation between ZFPM2-AS1 expression and clinicopathological characteristics in these patients. As shown in Supplementary Table S1, high ZFPM2-AS1 expression was positively associated with tumor invasion depth $(P=0.002)$, poor differentiation $(P=0.001)$, increased tumor size $(P=$ $0.018)$, N stage $(P=0.002)$, and advanced TNM stage $(P=0.007)$. Kaplan-Meier analyses demonstrated that high expression of ZFPM2-AS1 was strongly associated with reduced overall survival (Fig. 1G) and disease-free survival (Fig. 1H) in these patients. Univariate and multivariate survival analysis using a Cox proportional hazards regression model demonstrated that ZFPM2-AS1 expression could be regarded as an independent predictor of overall survival in patients with gastric cancer; TNM stage was an independent predictor of it, as well (Supplementary Table S5).

\section{Effects of ZFPM2-AS1 expression on the proliferation and apoptosis of gastric cancer cells in vitro and gastric tumor growth in vivo}

To evaluate the biofunctional roles of ZFPM2-AS1 expression in gastric cancer, we used shRNA-mediated knockdown and plasmid-mediated overexpression to exogenously manipulate expression of ZFPM2-AS1 in both MKN-45 and AGS cells (Supplementary Fig. S5A). A Cell Counting Kit - 8 assay and colony-forming assays demonstrated that knockdown of ZFPM2-AS1 expression markedly impaired gastric cancer cell proliferation (Fig. 2A and B), whereas ZFPM2-AS1 overexpression did the opposite (Supplementary Fig. S5B and C). EdU incorporation assays yielded similar results, demonstrating that downregulation of ZFPM2-AS1 expression was associated with reduced DNA synthetic activity (Fig. 2C), whereas enforced ZFPM2-AS1 expression had a promoting effect (Supplementary Fig. S5D).

We performed flow cytometric analysis to further determine whether ZFPM2-AS1 is involved in gastric cancer cell proliferation as reflected by cell-cycle arrest and apoptosis. The results revealed that gastric cancer cells transfected with two ZFPM2-AS1-specific shRNAs (\#1 and \#2) underwent obvious cell-cycle arrest at G0/G1 phase and that the population of cells at S phase decreased (Fig. 2D), whereas ZFPM2-AS1 overexpression had the opposite effects (Supplementary Fig. S5D). These findings were consistent with those of the EdU incorporation assays. Apoptosis assays revealed that the fraction of apoptotic cells was considerably higher among the ZFPM2-AS1-downregulated gastric cancer cells than in control cells (Fig. 2E).

To confirm the effect of ZFPM2-AS1 on gastric tumorigenesis in vivo, we subcutaneously injected AGS cells stably transfected with shRNA-ZFPM2-AS1 (shZFPM2-AS1) or nontargeting control shRNAand ZFPM2-AS1 or a control vector into the left thighs of nude mice. We found that ZFPM2-AS1-overexpressing tumor xenografts exhibited considerably greater growth than did by control xenografts, as demonstrated by increased mean tumor volumes and weights (Fig. 3A1, 3B1, and 3C1), whereas ZFPM2-AS1-downregulated 
tumor xenografts exhibited considerably less growth than did control xenografts (Fig. 3A2, 3B2, and 3C2). Immunohistochemical assays demonstrated that the Ki67 proliferation index was lower in the shZFPM2-AS1-transfected tumors and higher in the ZFPM2-AS1transfected tumors than in those in the control group (Fig. 3D). Taken together, these data indicated an important role for ZFPM2-AS1 in promoting gastric cancer cell proliferation in vitro and gastric tumor growth in vivo.

\section{ZFPM2-AS1 negatively regulates p53 protein expression and p53 downstream molecules}

To identify the molecular mechanism by which ZFPM2-AS1 exerts its effect on gastric cancer progression, we examined several signaling transduction pathways previously shown to be involved in unlimited proliferation and defective apoptosis of cancer cells ${ }^{18-20}$. We found that enforced ZFPM2-AS1 expression led to decreased p53 expression in AGS cells (Fig. 4A). In contrast, knockdown of ZFPM2-AS1 expression activated p53 expression in MKN-45 cells. We further examined several p53 downstream target genes. We found that expression of p21 and PUMA was downregulated and that of cyclin D1 and cyclin E1 was upregulated in ZFPM2-AS1-overexpressing AGS cells, whereas the opposite occurred in MKN-45 cells transfected with shZFPM2-AS1. In addition, we found that the p53 mRNA expression level did not change after dysregulated ZFPM2-AS1-based treatment in gastric cancer cells (Fig. 4B). Functionally, Cell Counting Kit - 8 and apoptotic assays demonstrated that downregulation of p53 expression partially abrogated the shZFPM2-AS1induced inhibitory effect on proliferation (Fig. 4C) and promoting effect on apoptosis of gastric cancer cells (Fig. 4D). However, of note is that AGS and MKN-45 cells do not contain any p53 mutations. We further examined the impact of ZFPM2-AS1 on mutant p53 expression in BGC-823 and MGC-803 cell lines carrying the p53 mutation. As shown in Supplementary Fig. S6A and B, knockdown of ZFPM2-AS1 did not affect the amount of endogenous mutant $\mathrm{p} 53$ protein. To determine whether ZFPM2-AS1 directly affected p53 protein expression, we performed an RNA pull-down assay. However, we did not find that p53 bound to ZFPM2-AS1 in our Western blot analysis. We hypothesized that ZFPM2-AS1 binds to an RNA-binding protein that serves as an adaptor protein and mediates the effect of ZFPM2-AS1. These data suggested that ZFPM2-AS1 inhibits the p53 signaling pathway to promote proliferation and suppress apoptosis of gastric cancer cells by indirectly regulating p53 protein expression.

\section{ZFPM2-AS1 physically binds to MIF protein}

To identify a possible mediator bridging ZFPM2-AS1 and to p53, we first examined whether manipulation of ZFPM2-AS1 expression affects the mRNA expression levels for its in cis genes. As shown in Supplementary Fig. S7A, no genes neighboring (e.g., ABRA, ANGPT1, LRP12, OXR1, ZFPM2) were changed along with ZFPM2-AS1. Although most of the transcript sequence of ZFPM2-AS1 overlaps in antisense the intron of the ZFPM2 proteincoding gene, we neither found any significant changes in ZFPM2 protein expression in ZFPM2-AS1-dysregulated AGS cells (data not shown). Furthermore, alteration of its neighboring genes did not impact ZFPM2-AS1 expression (Supplementary Fig. S7B-F). All of these data suggested that ZFPM2-AS1 acts in trans to inhibit p53 signaling in gastric cancer. 
We further performed RNA pull-down assays using a biotin-labeled ZFPM2-AS1 RNA probe to identify potential proteins associated with ZFPM2-AS1 in AGS cells. We analyzed RNA-associated proteins using sodium dodecyl sulfate-polyacrylamide gel electrophoresis and silver staining. We excised a unique band between 10 and $17 \mathrm{kDa}$ specific to ZFPM2AS1 and subjected it to mass spectrometry (Fig. 5A). This analysis identified MIF as a binding target of ZFPM2-AS1 (Supplementary Table S6), which was further confirmed by Western blot in three independent RNA pull-down assays (Fig. 5B). We further verified the specificity of this interaction using RIP (Fig. 5C). Notably, deletion-mapping analysis identified that the $5^{\prime}$-end segment (1-760 nt) of ZFPM2-AS1 is required for its association with MIF (Fig. 5D). These analyses confirmed that ZFPM2-AS1 physically binds to MIF in vitro. Consistent with previous reports, we found that the expression of MIF mRNA and protein in gastric cancer specimens was markedly higher than that in normal matched gastric tissue specimens (Supplementary Fig. S8A and B). To examine the correlation of ZFPM2AS1 and MIF expression, we sequentially examined the correlation of ZFPM2-AS1 expression with MIF mRNA and protein expression in 26 gastric cancer cases (cohort 2). We observed that ZFPM2-AS1 was positively correlated with the MIF protein level $(\mathrm{r}=0.773 ; P$ $<0.001)$ (Fig. 5E and F) but not with MIF mRNA level $(P=0.13)$ (Supplementary Fig. $\mathrm{S} 8 \mathrm{C})$. These data indicated that ZFPM2-AS1 may regulate MIF expression at the translational or posttranslational level.

\section{Inhibition of ZFPM2-AS1 expression destabilizes MIF protein}

To identify the mechanism of interaction between ZFPM2-AS1 and MIF, we first detected the subcellular location of ZFPM2-AS1 in gastric cancer cells. We found that ZFPM2-AS1 was present in both cytoplasmic and nuclear fractions of AGS cells (mean \pm standard deviation, $51.96 \% \pm 4.67 \%$ and $48.04 \% \pm 5.83 \%$, respectively) and MKN-45 cells (mean \pm standard deviation, $47.41 \% \pm 4.32 \%$ and $52.59 \% \pm 3.83 \%$, respectively) (Fig. 5G). Next, we explored the regulatory effect of ZFPM2-AS1 on MIF expression. We found significant upregulation of MIF protein expression upon ZFPM2-AS1 overexpression and downregulation of MIF protein expression upon ZFPM2-AS1 knockdown in gastric cancer cells (Fig. 5H). However, nochanges of MIF at the mRNA level were detected along with up- or down-regulation of ZFPM2-AS1 (Fig. 5I), further suggesting that ZFPM2-AS1 elevates MIF expression at the translational or posttranslational level. As shown in Fig. 5J, MIF protein levels in AGS and MKN-45 cells remained stable after treatment with cycloheximide (CHX) for 8 hours. Notably, treatment with the proteasome inhibitor MG132 over 8 hours did not increase MIF levels in gastric cancer cells (Fig. 5K). Since MIF could be stabilized by physical association with other component ${ }^{21}$, we hypothesized if ZFPM2AS1 could protect MIF through a similar mechanism. Indeed, MG132 treatment successfully blocked MIF destabilization with inhibition of ZFPM2-AS1 in gastric cancer cells (Fig. 5K). Functional studies demonstrated that knockdown of MIF expression abrogated ZFPM2-AS1 upregulation-induced promotion of proliferation (Supplementary Fig. S8D and E) and changes in the proportion of apoptotic gastric cancer cells (Supplementary Fig. S8F). Consistent with findings in GC cells, we also found altered expression of ZFPM2-AS1 could change MIF protein expression in colorectal cancer cells (Supplementary Fig. S9A and B). Taken together, these data provided evidence that ZFPM2-AS1 activates MIF expression at 
least partially by protecting MIF protein stability, thereby promoting proliferation and suppressing apoptosis of gastric cancer cells.

\section{ZFPM2-AS1 regulates p53 expression and subcellular location via upregulation of MIF expression}

Previous studies suggested that by physically associating with p53, MIF suppresses p53 protein stability and prevents its nuclear translocation ${ }^{15,22}$. Having shown that ZFPM2-AS1 interacts with MIF, we hypothesized that ZFPM2-AS1 modulates the p53 signaling pathway in an MIF-dependent manner. First, we determined the p53 and MIF protein expression by IHC in the GC tissue specimens with wide type TP53 showing low or high level of ZFPM2AS1 (Supplementary Fig. S10A). Also, we confirmed that MIF bound to p53 in gastric cancer cells (Fig. 6A) and that upregulation or downregulation of MIF markedly changed the expression of p53 protein (Fig. 6B). Next, we examined the role of MIF in the ZFPM2-AS1regulated p53 pathway using Western blot and qRT-PCR analysis. As expected, dysregulated ZFPM2-AS1 considerably altered the mRNA and protein expression for molecules downstream of p53 in gastric cancer cells; this effect could be partially rescued by MIF (Fig. $6 \mathrm{C}$ and D). By using in vitro CoIP assay, we determined that ZFPM2-AS1 is not required for the p53/MIF interaction (Supplementary Fig. S10B).

Furthermore, to determine whether ZFPM2-AS1 decreases the nuclear localization of p53 via MIF, we altered the expression of ZFPM2-AS1 in AGS and MKN-45 cells. As shown in Fig. 6E, ZFPM2-AS1 overexpression inhibited the nuclear translocation of p53. We also assessed whether MIF is necessary for ZFPM2-AS1-regulated nuclear translocation of p53 using Western blot analysis. Knockdown of MIF partially rescued the p53 nuclear translocation inhibited by ZFPM2-AS1 (Fig. 6F). Collectively, our data demonstrated that ZFPM2-AS1 promotes proliferation and suppresses apoptosis of gastric cancer cells by serving as an important upstream regulator of the MIF/p53 axis (Fig. 7).

\section{Discussion}

In the present study, we identified IncRNA ZFPM2-AS1 as a candidate oncogene involved in gastric cancer progression based on data from the Gene Expression Omnibus and TCGA data sets. For functional validation, we found that ZFPM2-AS1 expression was frequently upregulated in gastric cancer specimens and that this upregulated expression correlated with clinical aggressiveness and predicted poor survival. Moreover, ZFPM2-AS1 promoted proliferation of and inhibited cell-cycle arrest in and apoptosis of gastric cancer cells. Mechanistically, ZFPM2-AS1 inactivated the p53 signaling pathway by physically interacting with MIF and upregulating its expression in gastric cancer cells. Therefore, our clinical and experimental evidence strongly established that ZFPM2-AS1 is an oncogene and regulates 553 function, thus forming a ZFPM2-AS1/MIP/p53 signaling axis and promoting gastric cancer development and progression.

Because of the unlimited proliferation anddefective apoptosis of cancer cells, cancer therapy remains a major challenge. Recently, studies have increasingly identified many lncRNAs aberrantly expressed in tumor cells during gastric tumorigenesis. Dysregulatied lncRNAs may affect genetic or epigenetic communication and lead to a cellular growth advantage, 
resulting in progressive tumor growth ${ }^{8,23}$. Therefore, we conducted a series of experiments to characterize the novel oncogenic lncRNA ZFPM2-AS1 in gastric cancer, assess its possible relationship with gastric cancer, and explore the potential application of ZFPM2AS1in clinical management for gastric cancer.

ZFPM2-AS1 is located on chromosome 8q23, and three transcripts of the ZFPM2-AS1 gene are annotated in the National Center for Biotechnology Information database. However, the expression pattern and biological function of ZFPM2-AS1 in gastric cancer have yet to be reported. By using RACE assays, we identified a novel transcript of ZFPM2-AS1 different from those identified online. We demonstrated that ZFPM2-AS1 expression was frequently higher in gastric cancer specimens than in corresponding noncancerous tissue specimens. Importantly, ZFPM2-AS1 expression was positively correlated with depth of tumor invasion, differentiation grade, tumor size, $\mathrm{N}$ stage, and TNM stage, suggesting that ZFPM2-AS1 is a potential diagnostic biomarker and/or therapeutic target for gastric cancer. Also, patients with higher ZFPM2-AS1 expression exhibited poorer overall and disease-free survival. Further multivariate analysis revealed that the ZFPM2-AS1 expression level is a potential independent risk factor for overall survival after surgery, illustrating a prognostic value of ZFPM2-AS1 for gastric cancer. Role of ZFPM2-AS1 in gastric cancer was further investigated by examining alterations of biological behavior in gastric cancer cell lines with altered ZFPM2-AS1 expression. By applying loss- and gain-of-function approaches, we identified that ZFPM2-AS1 promotes cell proliferation concomitant with its induction of cell-cycle progression and apoptosis inability. Furthermore, we found that ZFPM2-AS1's impact on cancer cell proliferation and apoptosis was mediated by p53 signaling pathway. However, the detailed regulatory mechanism bridging ZFPM2-AS1 and p53 was yet to be determined.

By regulating gene expression via different mechanisms, lncRNAs play crucial roles in multiple biological processes ${ }^{24-26}$. ZFPM2-AS1 is transcribed in the antisense orientation of in the protein-coding gene ZFPM2. Several studies reported close relation between upstream antisense transcription and its corresponding gene ${ }^{27-29}$. Therefore, we first examined whether ZFPM2-AS1 regulates the expression of ZFPM2 in gastric cancer cells. qRT-PCR and Western blot analysis did not detect a significant effect of ZFPM2-AS1 on expression of ZFPM2 or other neighboring genes. Knockdown of expression of these neighboring genes also failed to change ZFPM2-AS1 expression. Therefore, these findings suggested that ZFPM2-AS1 should act in trans by targeting distant genes. In the present study, our RNA pull-down and mass spectrometric analysis identified the physical binding of MIF to ZFPM2-AS1. We also found that forced expression of ZFPM2-AS1 induced MIF protein expression, whereas inhibited expression of ZFPM2-AS1 did the opposite. We observed that MIF protein, not mRNA, expression was closely correlated with ZFPM2-AS1 expression. Functional analysis demonstrated that inhibition of MIF expression partially abrogated ZFPM2-AS1-induced promotion of gastric cancer cell proliferation. These findings strongly support that ZFPM2-AS1 elevates MIF expression and regulates proliferation of gastric cancer cells partially in an MIF-dependent way.

MIF is a tumor promoter with critical roles in the inflammation-tumorigenesis axis ${ }^{30}$. Researchers found that MIF was highly stable with low protein turnover and that its 
overexpression in tumor cells was prominent in a variety of human cancers ${ }^{31-34}$. Increased intratumoral MIF levels have correlated with clinical aggressiveness of cancer, implicating that MIF expression has a role in poor prognosis. However, treatment with proteasome inhibitor failed to increase MIF protein expression in a variety of cancers. The molecular mechanism stabilizing MIF protein in tumors cells is poorly uncovered. Schulz et al. ${ }^{21}$ reported that pharmacological inhibition of heat shock protein 90 activity or siRNAmediated knockdown of heat shock protein 90 expression destabilized MIF in a variety of human cancer cells, and this was mediated by the heat shock protein 90 -associated E3 ubiquitin ligase CHIP. Accumulating evidence demonstrated that hypoxia induces MIF expression or secretion in human cancer cells ${ }^{35}$. Additionally, authors reported MIF transcription was induced by hypoxia-inducible factor-1a (Hif1-a) ${ }^{36,37}$ and, conversely, that hypoxia-inducible factor-1a protein expression levels were stabilized by MIF. This raises the intriguing possible existence of MIF/Hif1-a positive feedback loop and these hypoxic tumors should be exquisitely sensitive to ZFPM2-AS1 inhibition.

LncRNAs, together with specific proteins, perform various functions depending on their subcellular location. Nuclear lncRNAs is primarily involved in the guidance and recruitment of histone protein modification enzymes and transcription factors to specific genomic loci, resulting in inactivation of tumor suppressors and activation of oncogenes ${ }^{38}$. Cytoplasmic lncRNAs frequently participate in cellular biological processes by functioning as competing endogenous RNAs or regulating mRNA or protein stability or its subcellular localization ${ }^{39}$. In this study, we found that a large proportion of MIF and ZFPM2-AS1 were located in the cytoplasm of gastric cancer cells. Since no proteins in the nucleus was identified to bind to ZFPM2-AS1, analyzing the ZFPM2-AS1-involved regulatory mechanism in the nucleus was expected to be explored in future studies.. In this study, since no MIF mRNA expression changed after induction of ZFPM2-AS1 expression, we therefore hypothesized if ZFPM2AS1 could protect MIF protein stabilization, which was a well characterized mechanism of IncRNA-protein interaction in the cytoplasm.. In accordance with findings reported previously, proteasome inhibitor MG132 failed to increase MIF levels in gastric cancer cells. However, upon ZFPM2-AS1 knockdown, MIF protein level increased along with treatment with MG132. The rapid decrease of MIF protein after ZFPM2-AS1 inhibition indicates that it is subject to proteasomal degradation under such circumstances. These results collectively showed that ZFPM2-AS1-induced accumulation of MIF protein is not resulted from increased protein synthesis, but from increased protein stability in gastric cancer cells. However, identifying the underlying mechanisms of ZFPM2-AS1's regulation of ubiquitination and/or ubiquitination-associated enzymes requires further investigation.

Upstream investigations of p53 signaling characterized MIF to be one of the most important regulators in p53-regulated tumor cell proliferation and apoptosis. By directly binding to p53, MIF contributes to destabilize p53 protein, and prevent translocation of p53 from the cytoplasm to the nucleus $15,22,40-43$. However, effective method to block this pro-tumor $\mathrm{MIF} / \mathrm{p} 53$ axis yet to be identified. As a key tumor suppressor, $\mathrm{p} 53$ regulates multiple critical biological processes, including apoptosis, cell-cycle arrest, and DNA repair. Inactivation of p53 is one of the most common events in gastric cancer progression. Genetic and epigenetic alterations causing inactivation of p53 are implicated to occur in about $50 \%$ of gastric cancer patients 44,45 . Notably, gastric cancer specimens obtained from $50 \%$ of patients exhibited 
p53 protein-positive immunostaining. Nevertheless, investigators later showed that the positive staining was largely attributable to expression of mutant $\mathrm{p} 53$, whereas expression of wild-type $\mathrm{p} 53$ protein became undetectable or lost in gastric tumors with the wild-type p53 allele ${ }^{46}$. Therefore, re-activation of the p53 pathway is an attractive strategy for pharmaceutical interference in tumor initiation and progression. Given the crucial roles of p53 in gastric cancer, researchers attempted to reactive this anti-tumor signaling by exploring its upstream regulators, including lncRNAs. For example, lncRNA-p21 represses the expression of many p53-targeted genes ${ }^{47,48}$. Also, the lncRNA PANDA inhibits the expression of several important proapoptotic genes upon DNA damage, and such effect is p53-dependent ${ }^{49}$. Depletion of the lncRNA MALAT1, results in activation of p53 and its downstream target genes ${ }^{50}$. Herein we demonstrated that posttranscriptional regulation of p53 expression is partly mediated by ZFPM2-AS1-induced MIF stability in the cytoplasm, thus controlling the expression of p53 target genes, facilitating gastric cancer cell proliferation, and inhibiting apoptosis. These results established a new molecular link between regulatory lncRNAs and p53 signaling.

In conclusion, we characterized the lncRNA ZFPM2-AS1 as a novel oncogene in gastric cancer patients. ZFPM2-AS1 expression was positively correlated with increased clinical aggressiveness of and poor prognosis for gastric cancer. By physically interacting with MIF, ZFPM2-AS1 protects MIF from degradation and increases MIF expression. The newly identified ZFPM2-AS1/MIF/p53 signaling axis exhibited significant protumor function in gastric cancer cases. ZFPM2-AS1 may be a promising prognostic predictor for gastric cancer, and the ZFPM2-AS1/MIF/p53 axis may be an effective therapeutic target for this cancer.

\section{Materials and Methods}

\section{Bioinformatic analysis}

Gene expression data and corresponding clinical data on gastric cancer patients were downloaded from TCGA and the publicly available Gene Expression Omnibus database using accession number GSE1279.

Patients

Gastric cancer specimens were collected from patients at Changhai Hospital, Second Military Medical University. No patient received preoperative treatment in this cohort and every patient signed for a written informed consent (cohort $1, n=73$ ). The tissue specimens were frozen in liquid nitrogen and all specimens were examined histologically. A tissue microarray was constructed using gastric cancer specimens from another patient cohort (cohort 2, $n=26$ ). Clinical information on the patients in these two cohorts are listed in Supplementary Tables S1 and S2.

\section{Cell culture}

HEK 239T; the nontumorigenic human gastric cell line GES-1; the gastric cancer cell lines BGC-823, MKN-28, MKN-45, MGC-803, and SGC-7901; and other cancer cell lines were purchased from the Committee of Type Culture Collection of the Chinese Academy of 
Sciences or the ATCC. Cell lines used in this study were obtained in 2015 and authenticated in August 2017 using short tandem repeat analysis. Cells were cultured at $37^{\circ} \mathrm{C}$ in a humidified incubator with an atmosphere of $5 \% \mathrm{CO}_{2}$ in Dulbecco's modified Eagle's medium supplemented with $10 \%$ fetal bovine serum.

\section{$5^{\prime}$ and $3^{\prime}$ rapid amplification of cDNA ends}

Total RNA was isolated by the RNeasy Plus Mini Kit (QIAGEN) according to the manufacturer's instructions. A SMARTer RACE 5'/3' Kit (Clontech Laboratories) was subsequently used to perform the rapid amplification of cDNA ends (RACE) experiments. Specific primers used in the report are listed in Supplementary Table S3.

\section{Quantitative reverse transcription-polymerase chain reaction and subcellular fractionation analysis}

Total RNA was extracted from the tissue specimens or cell lines using TRIzol reagent (Invitrogen). Random primers and a PrimeScript RT Reagent Kit (TaKaRa Bio, Shiga, Japan) were used to synthesize cDNA. Quantitative reverse transcription (RT)-polymerase chain reaction (PCR) analyses was performed using SYBR Green Master Mix (TaKaRa Bio) and further carried out on a LightCycler 480 Instrument II (Roche Diagnostics, Mannheim, Germany). The optimized PCR primers used are listed in Supplementary Table S3. PARIS

Kit (Life Technologies) was used to separate nuclear and cytosolic fractions of gastric cancer cellsaccording to the manufacturer's instructions.

\section{Transfection of plasmids and RNA interference}

Full-length (1-1168 bp) human ZFPM2-AS1 complementary DNA was cloned into a pCDH vector (Sigma-Aldrich). The ZFPM2-AS1 deletion mutant (ZDM) plasmids were constructed from $761 \mathrm{bp}$ and $941 \mathrm{bp}$ to $1168 \mathrm{bp}$ (ZDM\#1 and ZDM\#2). For lentiviral packaging, the plasmid/vector control pCDH-ZFPM-AS1 and packaging plasmids psPAX2 and PMD2.G (System Biosciences) were co-transfected into HEK 293T cells using Lipofectamine 2000 (Invitrogen). Also, short hairpin RNAs (shRNAs; Biotend Biotechnology), the packaging plasmids pPACK-GAG and pPACK-REV (System Biosciences), and the envelope plasmid pVSV-G (System Biosciences) were co-transfected into HEK 293T cells using the same regent. Viruses were harvested for 48 hours, filtered, and stored at $-80^{\circ} \mathrm{C}$ before use. An MIF-overexpressing plasmid (V5-tagged MIF vector) was purchased from Asia-Vector Biotechnology (Shanghai, People's Republic of China). Gastric cancer cells were seeded in a six-well plate. At confluence of 50-80\%, cells were infected with $1 \times 10^{6}$ recombinant lentivirus-transducing units and $6 \mu \mathrm{g} / \mathrm{mL}$ Polybrene (Sigma). For small interfering RNA (siRNA) infection, cells were seeded at 30-50\% confluence, and infected with 50-nM siRNAs using Lipofectamine 2000.

\section{Cell proliferation and colony formation assay}

Capability of gastric cell proliferation and colony formation were assessed as described previously ${ }^{51}$. 


\section{5-Ethynyl-2' -deoxyuridine proliferation assay}

A Cell-Light EdU Apollo 567 In Vitro Imaging Kit (RiboBio) was used to perform a 5ethynyl-2 ${ }^{\prime}$-deoxyuridine (EdU) proliferation assay as manufacturer suggested. In brief, $5 \times$ $10^{3}$ cells/well from indicated group were seeded into 96-well plates. Twenty-four hours after seeding, $100 \mu \mathrm{L}$ medium with $50 \mu \mathrm{M}$ EdU was added into each well. Cells were incubated for 2 hours at $37^{\circ} \mathrm{C}$. Subsequently, cells were fixed with $4 \%$ paraformaldehyde, and then stained with a Hoechst and Apollo reaction cocktail. A fluorescence microscope was used to capture the images Afterward, ratio of EdU-positive cells in total cells within each field were calculated.

\section{Flow cytometry}

Gastric cancer cells exposed to indicated treatment were stained with annex in V and propidium iodide (PI) from Apoptosis Detection Kit (BD Biosciences). Flow cytometry was then performed. Cell-cycle distribution and ratio of apoptotic cells from each group were counted and compared.

\section{Animals and tumor growth}

Female pathogen-free nude mice (5-week-old female nude mice with body weight of 18-22 g) were purchased from the National Cancer Institute. The animals were maintained in facilities approved by AAALAC International according to regulations and standards of the U.S. Department of Agriculture and Department of Health and Human Services. Five mice were arranged for each experiment without special randomization and blinding method. $100 \mu \mathrm{L}$ Hank's balanced salt solution with $1 \times 10^{6}$ gastric cancer cells were injected subcutaneously into the left thighs of the mice. The resulting tumors were measured every week. The mice were killed via cervical dislocation 35 days after injection, and their tumors were removed and weighed.

\section{Protein extraction and Western blotting}

Standard Western blotting procedure was performed using whole-cell protein lysates as described elsewhere. ${ }^{52}$ Detailed information on the antibodies is listed in Supplementary Table S4.

\section{RNA pull-down and mass spectrometry}

ZFPM2-AS1 and its antisense plasmid were linearly cut, transcribed, and biotin-labeled in vitro with Bio-16-UTP (Life Technologies) using a MAXI script T7 Transcription Kit (Life Technologies). Protein-RNA interactions were carried out using a Pierce Magnetic RNAProtein Pull-Down Kit (Life Technologies) with lysates of AGS and MKN-45 cells. Then, the proteins were detected using Western blot analysis or resolved via gradient gel electrophoresis followed by mass spectrometric identification.

\section{RNA immunoprecipitation, immunofluorescence and co-immunoprecipitation}

A Magna RIP RNA-Binding Protein Immunoprecipitation Kit (Millipore) was used to carry out RNA immunoprecipitation (RIP) experiments according to the manufacture's 
recommendation. The MIF-based RIP assays (Santa Cruz Biotechnology), immunofluorescence and co-immumoprecipitation were performed as reported previously.

\section{Immunohistochemical assay}

Immunohistochemical analyses of gastric cancer specimens were conducted with specific antibodies as described previously (16). Expression of proteins in the specimens was estimated according to the percentage and intensity of the stained tumor cells. The staining percentage and intensity were graded as $0(0-4 \%), 1$ (5-24\%), 2 (25-49\%), 3 (50-74\%), 4 ( $\geq 75 \%$ ), and $0,1,2$, or 3 , respectively. The final score was obtained by multiplication of percentage and intensity score. Tissue samples with final staining scores of $0,1-4,5-8$, and 9-12 were considered negative (-), weakly positive (+), moderately positive (++), and strongly positive (+++), respectively. All the sections were individually scored by two investigators who were blinded to the clinical information of the gastric cancer cases.

\section{Statistical analysis}

The significance of differences among covariates was determined with a two-tailed $t$ test or the Fisher exact test. All in vitro experiments were performed in triplicate and at least three times. Data were presented either as means \pm standard deviation from one representative independent experiment of three with similar results or means \pm standard error of the mean from three independent experiments. Student $t$-test (two-tailed) or one-way analysis of variance was used to determine the significance of the in vitro and in vivo data. In all the comparisons, statistical analysis was carried out by using SPSS software program (version 17.0; IBM Corporation) and $P<0.05$ were considered statistically significant

\section{Supplementary Material}

Refer to Web version on PubMed Central for supplementary material.

\section{Acknowledgments}

Financial Support: This work was supported by grants R01CA172233, R01CA195651, and R01CA198090 from the National Cancer Institute, National Institutes of Health (to K. Xie) and grant 81772640 from the National Natural Science Foundation of China (to X. Kong).

We would like to thank Don Norwood for editing the manuscript.

\section{References}

1. Sugano K. Screening of gastric cancer in Asia. Best practice \& research Clinical gastroenterology. 2015; 29:895-905. [PubMed: 26651251]

2. Siegel R, Naishadham D, Jemal A. Cancer statistics, 2013. CA: a cancer journal for clinicians. 2013; 63:11-30. [PubMed: 23335087]

3. Lin Y, Ueda J, Kikuchi S, Totsuka Y, Wei WQ, Qiao YL, et al. Comparative epidemiology of gastric cancer between Japan and China. World journal of gastroenterology : WJG. 2011; 17:4421-4428. [PubMed: 22110269]

4. Tan P, Yeoh KG. Genetics and Molecular Pathogenesis of Gastric Adenocarcinoma. Gastroenterology. 2015; 149:1153-1162. e1153. [PubMed: 26073375]

5. Comprehensive molecular characterization of gastric adenocarcinoma. Nature. 2014; 513:202-209. [PubMed: 25079317] 
6. Fatica A, Bozzoni I. Long non-coding RNAs: new players in cell differentiation and development. Nat Rev Genet. 2014; 15:7-21. [PubMed: 24296535]

7. Ulitsky I, Bartel DP. lincRNAs: genomics, evolution, and mechanisms. Cell. 2013; 154:26-46. [PubMed: 23827673]

8. Schmitt AM, Chang HY. Long Noncoding RNAs in Cancer Pathways. Cancer cell. 2016; 29:452463. [PubMed: 27070700]

9. Huarte M. The emerging role of lncRNAs in cancer. Nat Med. 2015; 21:1253-1261. [PubMed: 26540387]

10. Katayama S, Tomaru Y, Kasukawa T, Waki K, Nakanishi M, Nakamura M, et al. Antisense transcription in the mammalian transcriptome. Science. 2005; 309:1564-1566. [PubMed: 16141073]

11. Wight M, Werner A. The functions of natural antisense transcripts. Essays in biochemistry. 2013; 54:91-101. [PubMed: 23829529]

12. Werner A. Biological functions of natural antisense transcripts. BMC biology. 2013; 11:31. [PubMed: 23577602]

13. Villegas VE, Zaphiropoulos PG. Neighboring gene regulation by antisense long non-coding RNAs. International journal of molecular sciences. 2015; 16:3251-3266. [PubMed: 25654223]

14. Huang B, Song JH, Cheng Y, Abraham JM, Ibrahim S, Sun Z, et al. Long non-coding antisense RNA KRT7-AS is activated in gastric cancers and supports cancer cell progression by increasing KRT7 expression. Oncogene. 2016; 35:4927-4936. [PubMed: 26876208]

15. Fukaya R, Ohta S, Yaguchi T, Matsuzaki Y, Sugihara E, Okano H, et al. MIF Maintains the Tumorigenic Capacity of Brain Tumor-Initiating Cells by Directly Inhibiting p53. Cancer Res. 2016; 76:2813-2823. [PubMed: 26980763]

16. Shun CT, Lin JT, Huang SP, Lin MT, Wu MS. Expression of macrophage migration inhibitory factor is associated with enhanced angiogenesis and advanced stage in gastric carcinomas. World journal of gastroenterology : WJG. 2005; 11:3767-3771. [PubMed: 15968736]

17. Sun B, Nishihira J, Suzuki M, Fukushima N, Ishibashi T, Kondo M, et al. Induction of macrophage migration inhibitory factor by lysophosphatidic acid: relevance to tumor growth and angiogenesis. International journal of molecular medicine. 2003; 12:633-641. [PubMed: 12964047]

18. Cotter TG. Apoptosis and cancer: the genesis of a research field. Nat Rev Cancer. 2009; 9:501507. [PubMed: 19550425]

19. Lopez-Saez JF, de la Torre C, Pincheira J, Gimenez-Martin G. Cell proliferation and cancer. Histology and histopathology. 1998; 13:1197-1214. [PubMed: 9810511]

20. Pardal R, Molofsky AV, He S, Morrison SJ. Stem cell self-renewal and cancer cell proliferation are regulated by common networks that balance the activation of proto-oncogenes and tumor suppressors. Cold Spring Harbor symposia on quantitative biology. 2005; 70:177-185. [PubMed: 16869752]

21. Schulz R, Marchenko ND, Holembowski L, Fingerle-Rowson G, Pesic M, Zender L, et al. Inhibiting the HSP90 chaperone destabilizes macrophage migration inhibitory factor and thereby inhibits breast tumor progression. The Journal of experimental medicine. 2012; 209:275-289. [PubMed: 22271573]

22. Mitchell RA, Liao H, Chesney J, Fingerle-Rowson G, Baugh J, David J, et al. Macrophage migration inhibitory factor (MIF) sustains macrophage proinflammatory function by inhibiting p53: regulatory role in the innate immune response. Proc Natl Acad Sci U S A. 2002; 99:345-350. [PubMed: 11756671]

23. Beermann J, Piccoli MT, Viereck J, Thum T. Non-coding RNAs in Development and Disease: Background, Mechanisms, and Therapeutic Approaches. Physiological reviews. 2016; 96:12971325. [PubMed: 27535639]

24. Fu M, Zou C, Pan L, Liang W, Qian H, Xu W, et al. Long noncoding RNAs in digestive system cancers: Functional roles, molecular mechanisms, and clinical implications (Review). Oncology reports. 2016; 36:1207-1218. [PubMed: 27431376]

25. Li T, Mo X, Fu L, Xiao B, Guo J. Molecular mechanisms of long noncoding RNAs on gastric cancer. Oncotarget. 2016; 7:8601-8612. [PubMed: 26788991] 
26. Wang KC, Chang HY. Molecular mechanisms of long noncoding RNAs. Mol Cell. 2011; 43:904914. [PubMed: 21925379]

27. Zhang CL, Zhu KP, Ma XL. Antisense lncRNA FOXC2-AS1 promotes doxorubicin resistance in osteosarcoma by increasing the expression of FOXC2. Cancer letters. 2017; 396:66-75. [PubMed: 28323030]

28. Amit-Avraham I, Pozner G, Eshar S, Fastman Y, Kolevzon N, Yavin E, et al. Antisense long noncoding RNAs regulate var gene activation in the malaria parasite Plasmodium falciparum. Proc Natl Acad Sci U S A. 2015; 112:E982-991. [PubMed: 25691743]

29. Wei N, Wang Y, Xu RX, Wang GQ, Xiong Y, Yu TY, et al. PU. 1 antisense lncRNA against its mRNA translation promotes adipogenesis in porcine preadipocytes. Animal genetics. 2015; 46:133-140. [PubMed: 25691151]

30. Conroy H, Mawhinney L, Donnelly SC. Inflammation and cancer: macrophage migration inhibitory factor (MIF)--the potential missing link. QJM : monthly journal of the Association of Physicians. 2010; 103:831-836. [PubMed: 20805118]

31. He XX, Yang J, Ding YW, Liu W, Shen QY, Xia HH. Increased epithelial and serum expression of macrophage migration inhibitory factor (MIF) in gastric cancer: potential role of MIF in gastric carcinogenesis. Gut. 2006; 55:797-802. [PubMed: 16488898]

32. Yang S, He P, Wang J, Schetter A, Tang W, Funamizu N, et al. A Novel MIF Signaling Pathway Drives the Malignant Character of Pancreatic Cancer by Targeting NR3C2. Cancer Res. 2016; 76:3838-3850. [PubMed: 27197190]

33. Schulz R, Streller F, Scheel AH, Ruschoff J, Reinert MC, Dobbelstein M, et al. HER2/ErbB2 activates HSF1 and thereby controls HSP90 clients including MIF in HER2-overexpressing breast cancer. Cell death \& disease. 2014; 5:e980. [PubMed: 24384723]

34. Dessein AF, Stechly L, Jonckheere N, Dumont P, Monte D, Leteurtre E, et al. Autocrine induction of invasive and metastatic phenotypes by the MIF-CXCR4 axis in drug-resistant human colon cancer cells. Cancer Res. 2010; 70:4644-4654. [PubMed: 20460542]

35. Larsen M, Tazzyman S, Lund EL, Junker N, Lewis CE, Kristjansen PE, et al. Hypoxia-induced secretion of macrophage migration-inhibitory factor from MCF-7 breast cancer cells is regulated in a hypoxia-inducible factor-independent manner. Cancer letters. 2008; 265:239-249. [PubMed: 18353538]

36. Oda S, Oda T, Nishi K, Takabuchi S, Wakamatsu T, Tanaka T, et al. Macrophage migration inhibitory factor activates hypoxia-inducible factor in a p53-dependent manner. Plos One. 2008; 3:e2215. [PubMed: 18493321]

37. Winner M, Koong AC, Rendon BE, Zundel W, Mitchell RA. Amplification of tumor hypoxic responses by macrophage migration inhibitory factor-dependent hypoxia-inducible factor stabilization. Cancer Res. 2007; 67:186-193. [PubMed: 17210698]

38. Rinn JL, Chang HY. Genome regulation by long noncoding RNAs. Annual review of biochemistry. 2012; 81:145-166.

39. Shi X, Sun M, Wu Y, Yao Y, Liu H, Wu G, et al. Post-transcriptional regulation of long noncoding RNAs in cancer. Tumour biology : the journal of the International Society for Oncodevelopmental Biology and Medicine. 2015; 36:503-513. [PubMed: 25618601]

40. Brock SE, Rendon BE, Xin D, Yaddanapudi K, Mitchell RA. MIF family members cooperatively inhibit p53 expression and activity. Plos One. 2014; 9:e99795. [PubMed: 24932684]

41. Salminen A, Kaarniranta K. Control of p53 and NF-kappaB signaling by WIP1 and MIF: role in cellular senescence and organismal aging. Cellular signalling. 2011; 23:747-752. [PubMed: 20940041]

42. Jung H, Seong HA, Ha H. Direct interaction between NM23-H1 and macrophage migration inhibitory factor (MIF) is critical for alleviation of MIF-mediated suppression of p53 activity. $\mathrm{J}$ Biol Chem. 2008; 283:32669-32679. [PubMed: 18815136]

43. Jung H, Seong HA, Ha H. Critical role of cysteine residue 81 of macrophage migration inhibitory factor (MIF) in MIF-induced inhibition of p53 activity. J Biol Chem. 2008; 283:20383-20396. [PubMed: 18502749] 
44. Bellini MF, Cadamuro AC, Succi M, Proenca MA, Silva AE. Alterations of the TP53 gene in gastric and esophageal carcinogenesis. Journal of biomedicine \& biotechnology. 2012; 2012:891961. [PubMed: 22919278]

45. Imazeki F, Omata M, Nose H, Ohto M, Isono K. p53 gene mutations in gastric and esophageal cancers. Gastroenterology. 1992; 103:892-896. [PubMed: 1499939]

46. Gabbert HE, Muller W, Schneiders A, Meier S, Hommel G. The relationship of p53 expression to the prognosis of 418 patients with gastric carcinoma. Cancer. 1995; 76:720-726. [PubMed: 8625172]

47. Huarte M, Guttman M, Feldser D, Garber M, Koziol MJ, Kenzelmann-Broz D, et al. A large intergenic noncoding RNA induced by p53 mediates global gene repression in the p53 response. Cell. 2010; 142:409-419. [PubMed: 20673990]

48. Dimitrova N, Zamudio JR, Jong RM, Soukup D, Resnick R, Sarma K, et al. LincRNA-p21 activates p21 in cis to promote Polycomb target gene expression and to enforce the G1/S checkpoint. Molecular cell. 2014; 54:777-790. [PubMed: 24857549]

49. Hung T, Wang Y, Lin MF, Koegel AK, Kotake Y, Grant GD, et al. Extensive and coordinated transcription of noncoding RNAs within cell-cycle promoters. Nature genetics. 2011; 43:621-629. [PubMed: 21642992]

50. Tripathi V, Shen Z, Chakraborty A, Giri S, Freier SM, Wu X, et al. Long noncoding RNA MALAT1 controls cell cycle progression by regulating the expression of oncogenic transcription factor B-MYB. PLoS genetics. 2013; 9:e1003368. [PubMed: 23555285]

51. Guo K, Cui J, Quan M, Xie D, Jia Z, Wei D, et al. The Novel KLF4/MSI2 Signaling Pathway Regulates Growth and Metastasis of Pancreatic Cancer. Clin Cancer Res. 2017; 23:687-696. [PubMed: 27449499]

52. Kong F, Kong X, Du Y, Chen Y, Deng X, Zhu J, et al. STK33 Promotes Growth and Progression of Pancreatic Cancer as a Critical Downstream Mediator of HIF1alpha. Cancer Res. 2017; 77:68516862. [PubMed: 29038348] 


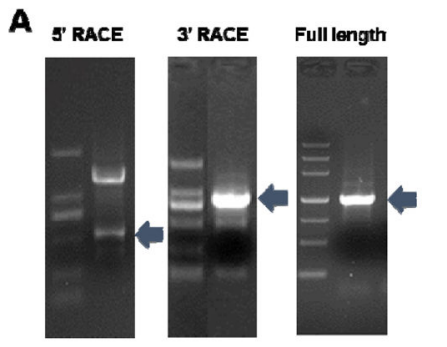

C

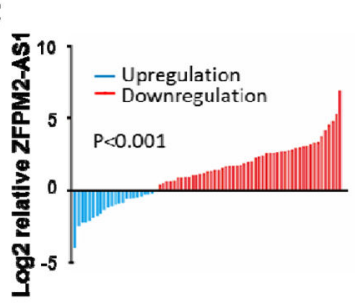

$\mathbf{F}$

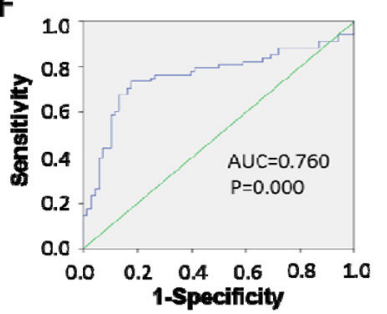

D

G
B
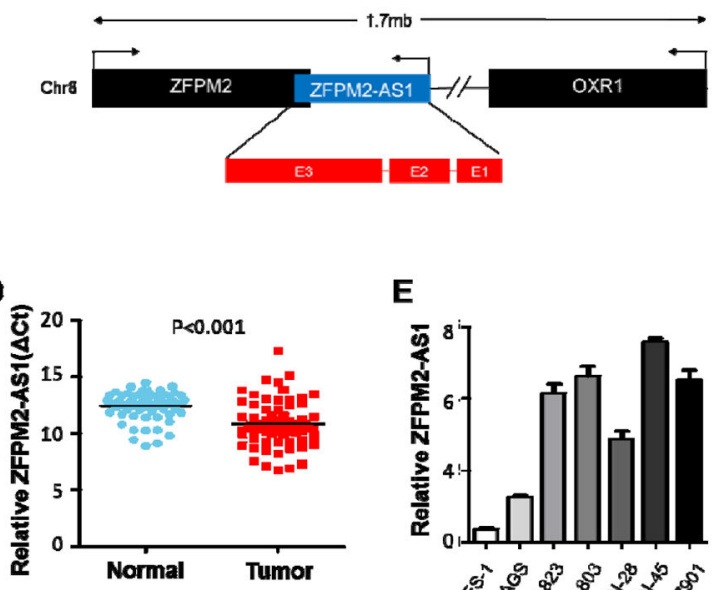

E
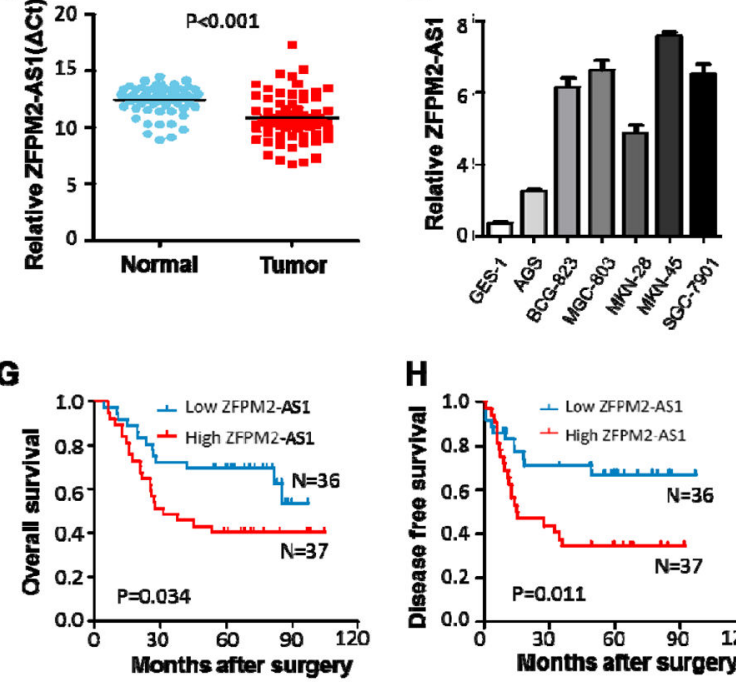

H

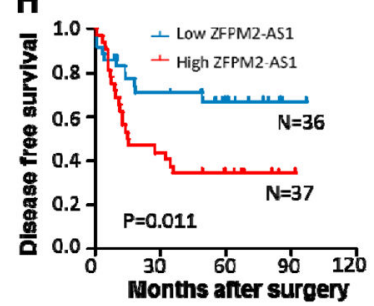

Figure 1. Upregulation of ZFPM2-AS1 expression predicts poor prognosis for gastric cancer A, 5', 3', and full-length RACE for ZFPM2-AS1. B, schematic of the location of ZFPM2AS1. The partial sequence of ZFPM2-AS1 overlaps in antisense the intron of the ZFPM2 protein-coding gene. C, the relative ZFPM2-AS1 expression levels in gastric cancer and adjacent nontumor gastric tissue specimens. The results were presented as $\log 2\left(2^{-\Delta \Delta C t}\right)$. D, ZFPM2-AS1 expression in gastric cancer and adjacent nontumor gastric tissue specimens ( $n$ = 73) was analyzed using qRT-PCR. The ZFPM2-AS1 expression level was normalized to that of U6. E, ZFPM2-AS1 expression levels in normal gastric cells (GES-1) and gastric cancer cell lines (AGS, BGC-823, MGC-803, MKN-28, MKN-45, and SGC-7901). F, receiver operating characteristic curve for prediction of gastric cancer according to qRTPCR-based ZFPM2-AS1 expression level. G and $\mathbf{H}$, Kaplan-Meier analysis of overall survival (OS) (G) and disease-free survival (DFS) (H) in gastric cancer patients. 
A
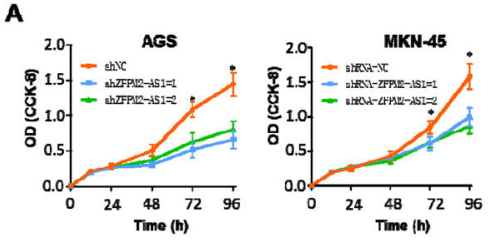

C
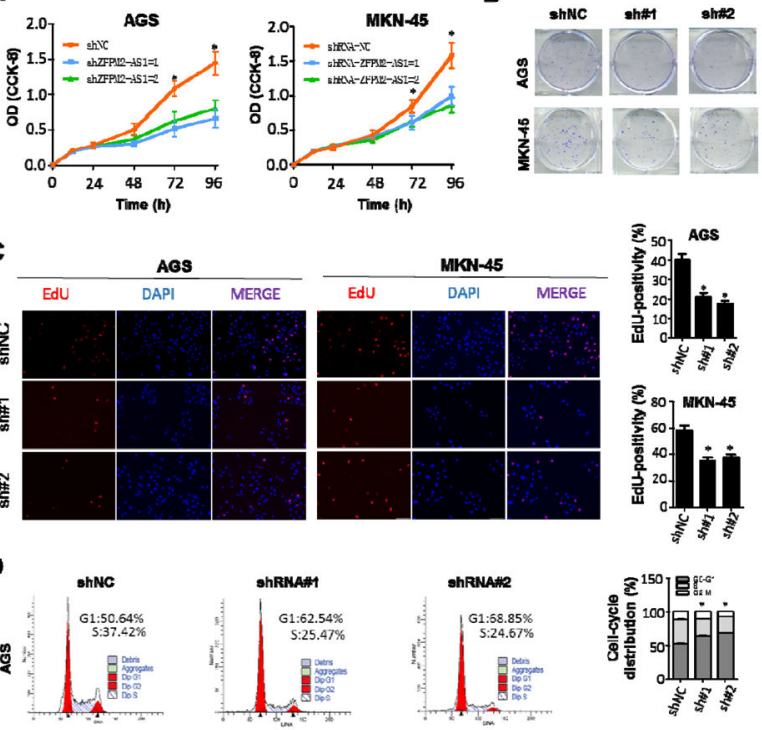

是

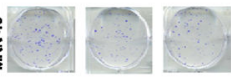

D
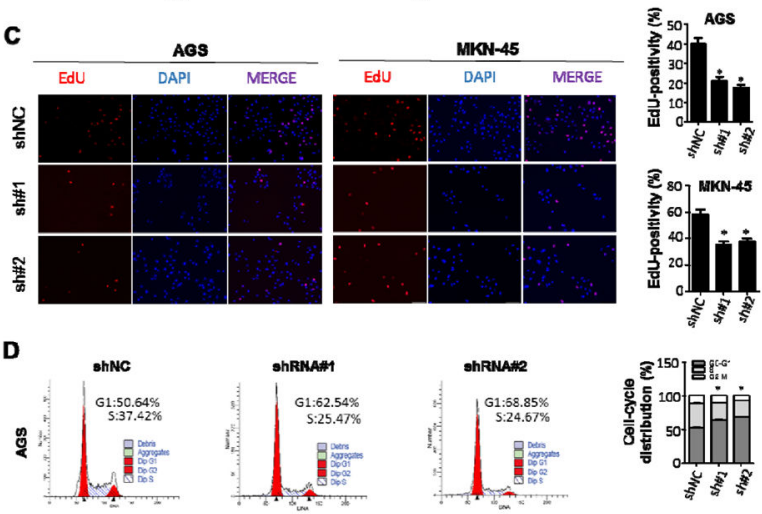
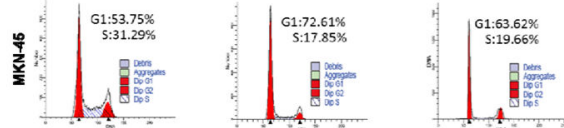

E
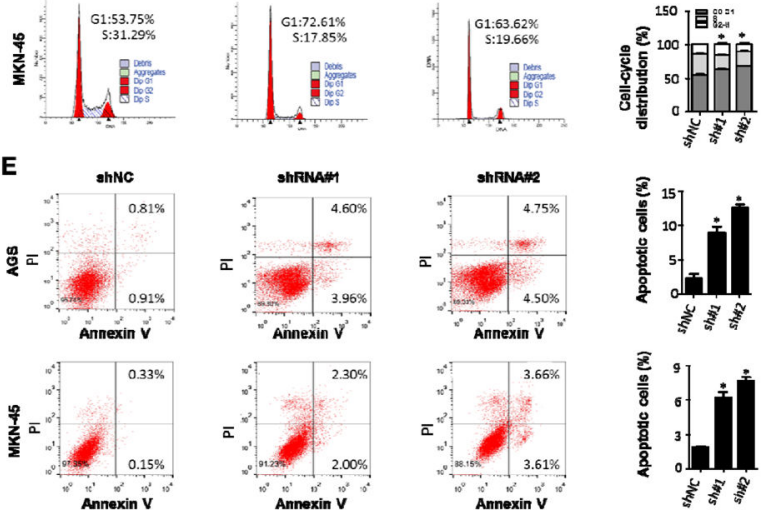

Figure 2. ZFPM2-AS1 promotes proliferation and suppresses apoptosis of gastric cancer cells in vitro

A, assessment of gastric cancer cell growth in vitro using a Cell Counting Kit - 8 (CCK-8) at the indicated time points. OD, optical density. $* P<0.05$. B, colony formation assay of AGS and MKN-45 cells transfected with ZFPM2-AS1-specific shRNAs (sh\#1 and sh\#2) or nontargeting control shRNA (shNC). C, EdU incorporation assay performed to determine the effect of ZFPM2-AS1 inhibition on DNA synthesis during gastric cancer cell proliferation. Knockdown of ZFPM2-AS1 decreases the ratio of EdU-positive (S phase) cells in total gastric cancer cells. D, flow cytometric analysis of cell-cycle arrest 48 hours after treatment with shZFPM2-AS1 in AGS and MKN-45 cells. $* P<0.05$. E, flow cytometric analysis of the rates of apoptotic AGS and MKN-45 cells after treatment with shZFPM2AS1 or shNC. $* P<0.05$. 

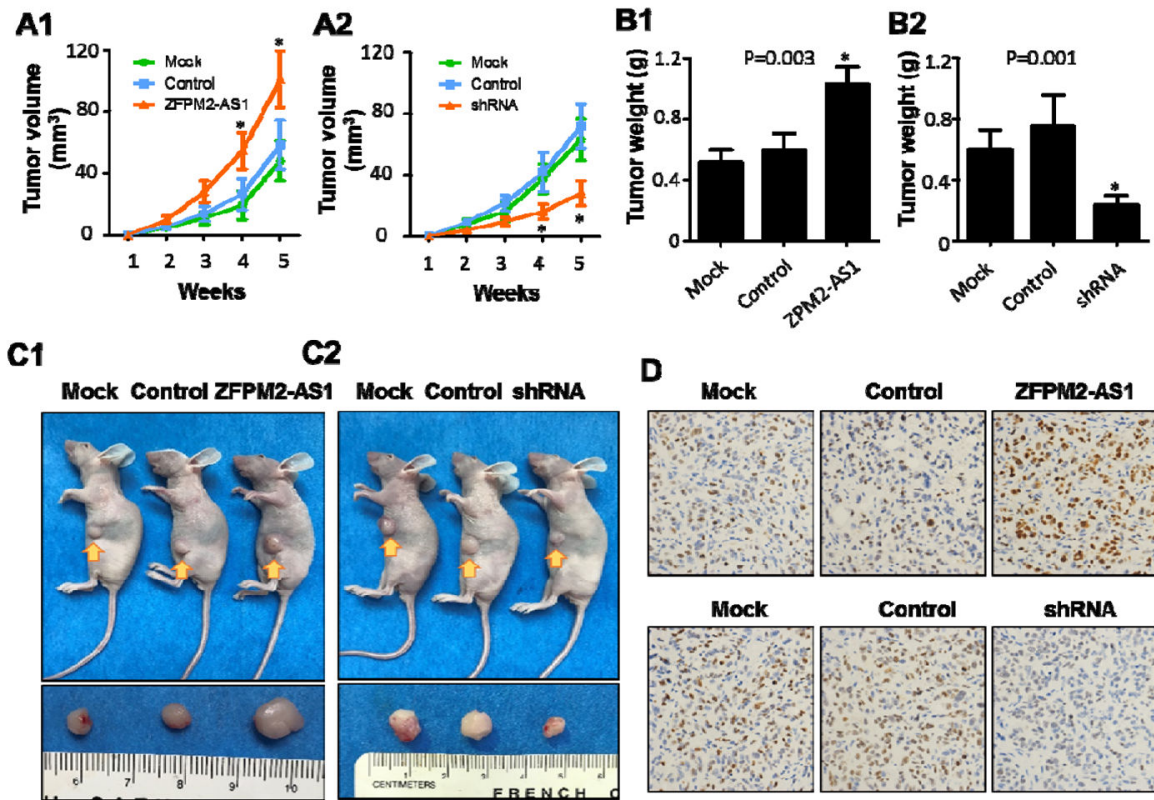

C2

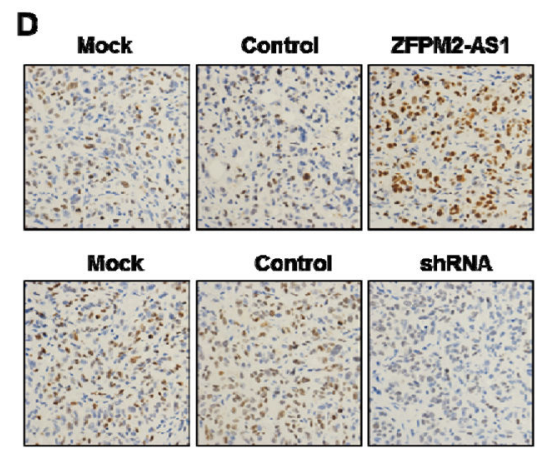

Figure 3. ZFPM2-AS1 promotes the growth of gastric cancer cells in vivo

ZFPM2-AS1-overexpressing plasmid- or shRNA-treated AGS cells were injected subcutaneously into the left thighs of nude mice $\left(1 \times 10^{6}\right.$ cells per mouse, five mice per group). Shown are tumor growth curves (A1 and A2; $* P<0.05)$, tumor weights (B1 and B2) and gross tumors $(\mathbf{C 1}$ and $\mathbf{C 2})$ in the indicated mouse groups. The formula used to calculate tumor volume was $\mathrm{ab}^{2} / 2$, with a representing the longest diameter of the tumor and $\mathrm{b}$ representing the diameter perpendicular to the longest one. D, representative images of immunohistochemical staining of tumor specimens for $\mathrm{Ki} 67$ in the indicated groups. 

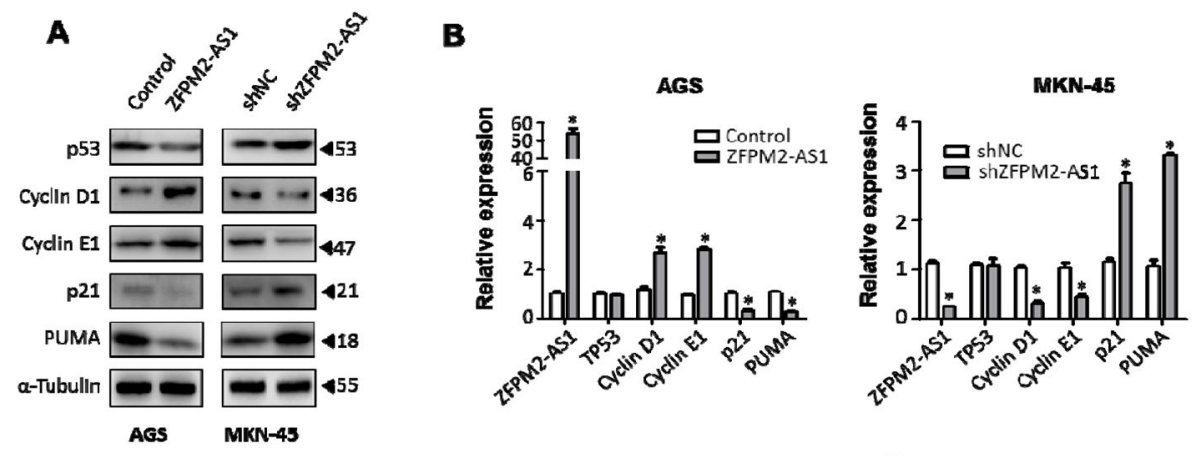

C
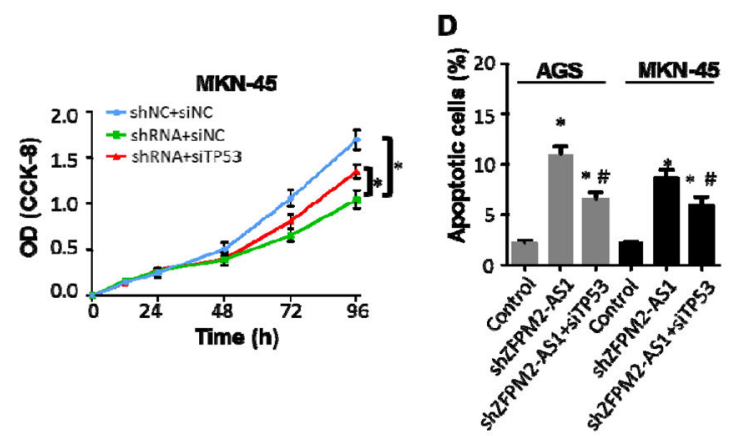

Figure 4. ZFPM2-AS1 negatively regulates expression of 553 protein and represses the expression of p53 downstream molecules

A, Western blot analysis demonstrating that upregulation or downregulation of ZFPM2-AS1 expression changed the expression of p53 and its downstream targets cyclin D1, cyclin E1, p21, and PUMA in gastric cancer cell lines. B, ZFPM2-AS1 knockdown altered the mRNA expression for p53 downstream genes but did not change the p53 mRNA expression level in gastric cancer cell lines. * $\mathrm{P}<0.05$. C, the growth rates for AGS and MKN-45 cells. *P < 0.05. D, apoptotic analysis of shZFPM2-AS1-transfected gastric cancer cells with or without siTp53. *Statistically significant when compared with the control group $(P<0.05)$; \#Statistically significant when compared with the shZFPM2-AS1 group $(P<0.05)$. 
A

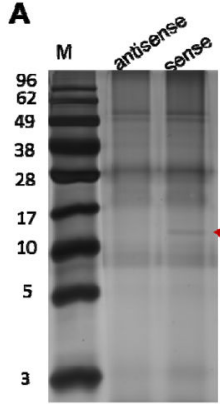

E

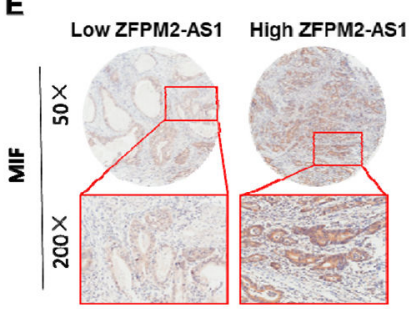

H

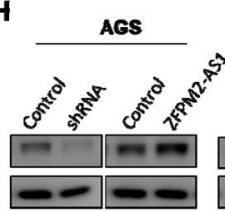

J

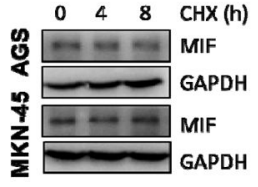

B

$\mathbf{K}$

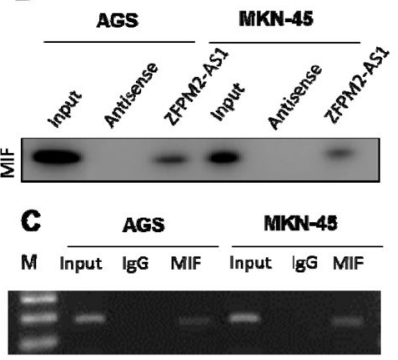

$F$

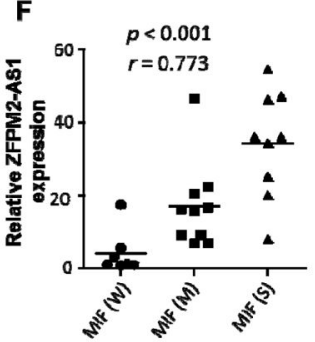

I
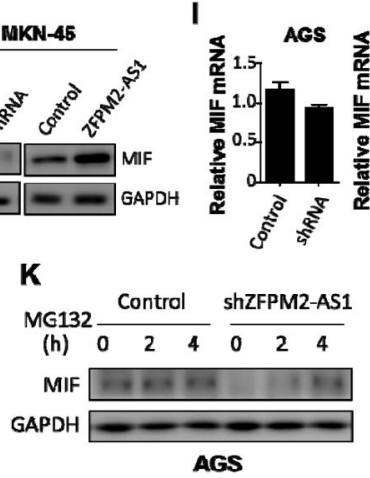

G

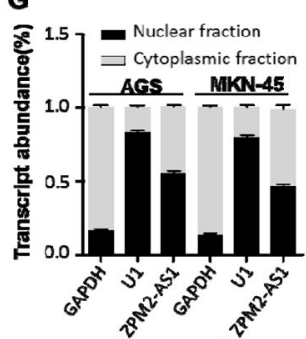

D
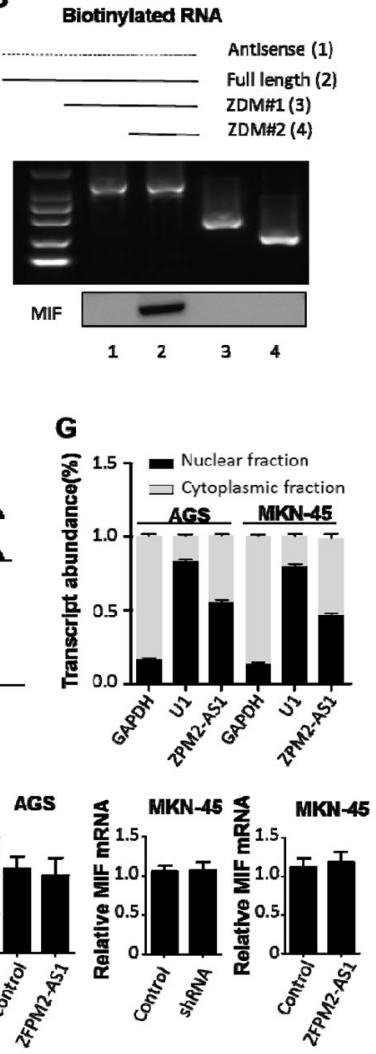

Control

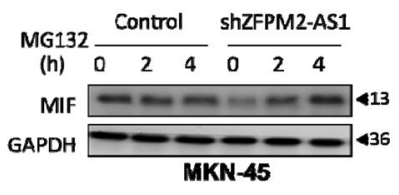

Figure 5. ZFPM2-AS1 binds to MIF and increases MIF expression

A, SDS-PAGE analysis with silver staining showed the immunoprecipitated proteins from an AGS cell pulled down by ZFPM2-AS1 or its antisense RNA. The arrow indicates the possible ZFPM2-AS1 bound protein subsequently excised for mass spectrometry. B, biotinylated ZFPM2-AS1 or antisense RNA was incubated with whole-cell protein lysates of AGS and MKN-45 cells in another RNA pull-down assay. The MIF protein was detected by Western blot using a specific MIF antibody. C, RIP experiments determined the interaction of MIF and ZFPM2-AS1. D, immunoblotting for AGS cells pulled down with different fragment of ZFPM2-AS1.. E, representative immunohistochemical images of MIF protein expression with high or low ZFPM2-AS1 expression in gastric cancer specimens $(n=26) . \mathbf{F}$, direct correlation between ZFPM2-AS1 and MIF protein expression as assessed using Spearman correlation coefficient analysis $(n=26)$. Expression of ZFPM2-AS1 and the immunohistochemistry score for MIF protein in each gastric cancer specimen were examined separately. W, weak; M, moderate; S, strong. G, intracellular distribution of ZFPM2-AS1 in AGS and MKN-45 cell lysates were analyzed by RT-qPCR. GAPDH and U6 were used as internal control. H, Western blot of MIF expression after decreased or increased ZFPM2-AS1 expression in AGS and MKN-45 cells. I, qRT-PCR results demonstrating that overexpression and knockdown of expression of ZFPM2-AS1 did not 
affect MIF mRNA expression levels in gastric cancer cells. J, Western blot of MIF in AGS and MKN-45 cells treated with $40 \mu \mathrm{g} / \mathrm{mL} \mathrm{CHX}$ for the indicated times. K, Western blots showing effect of proteasome inhibitor MG132 on MIF expression in gastric cancer cells with or without shZFPM2-AS1 treatment for 48 hours.. 
A

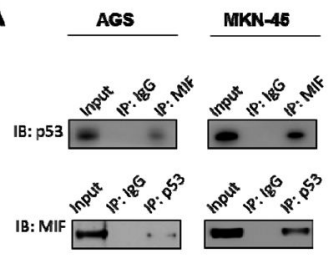

C

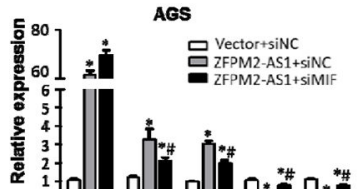

${ }_{0}^{1}$ 近
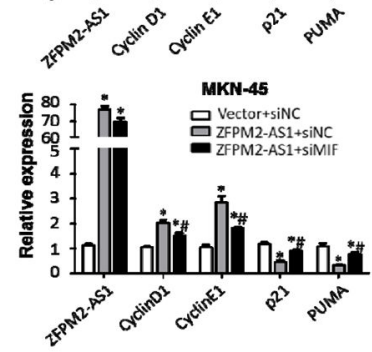

E
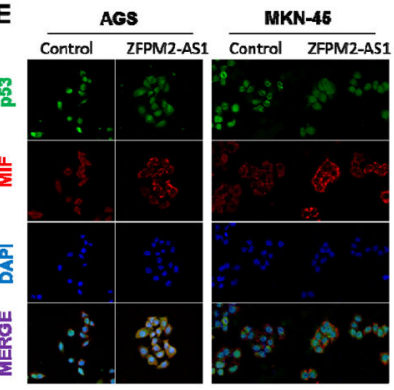

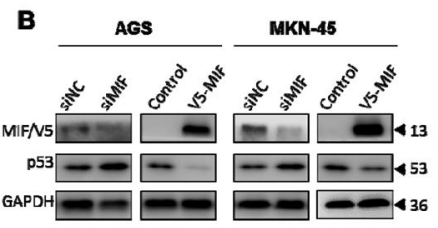

D

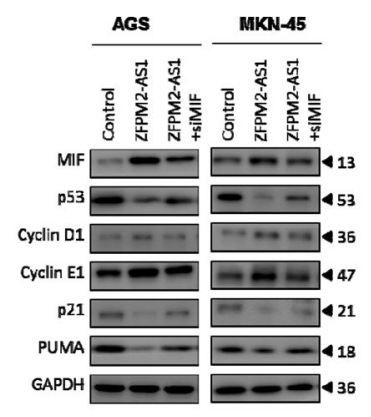

Figure 6. The ZFPM2-AS1/MIF axis promotes gastric cancer cell proliferation and suppresses their apoptosis by attenuating the $\mathbf{p 3}$ signaling

A, Western blots of extracts of AGS and MKN-45 cells were subjected to coimmunoprecipitation (IP) using an anti-MIF antibody or control IgG performed with an antip53 antibody. Reciprocal co-immunoprecipitation was performed using anti-p53 and -IgG antibodies followed by Western blotting with an anti-MIF antibody. IB, immunoblot. B, Western blot analysis of p53 expression in AGS and MKN-45 cells after decreased or increased MIF expression. C and D, qRT-PCR (C) and Western blot (D) analysis results demonstrating that ZFPM2-AS1 regulated the p53 pathway in an MIF-dependent manner. The mRNA and protein expression for the p53-targeting genes cyclin D1, cyclin E1, p21, and PUMA in the indicated groups were examined. GAPDH was used as a loading control. siMIF, MIF-specific siRNA. * $P<0.05$ for comparison between "Vector+siNC" and "ZFPM2AS1+siNC"; \#P<0.05 for comparison between "ZFPM2-AS1+siNC" and "ZFPM2-

AS1+siMIF". E, immunofluroescent stains of AGS and MKN-45 cells transfected with an ZFPM2-AS1-overexpressing vector and cultured in vitro for 48 hours for p53 (green), MIF (red), and nuclei (4',6-dimidino-2-phenylindole [DAPI]; blue). F, ZFPM2-AS1 was cotransfected with $50 \mathrm{nM}$ siMIF or a control siRNA (siNC) into AGS and MKN-45 cells for 48 hours. The Western blots show that ZFPM2-AS1 inhibited nuclear translocation of p53 by activating MIF expression. 


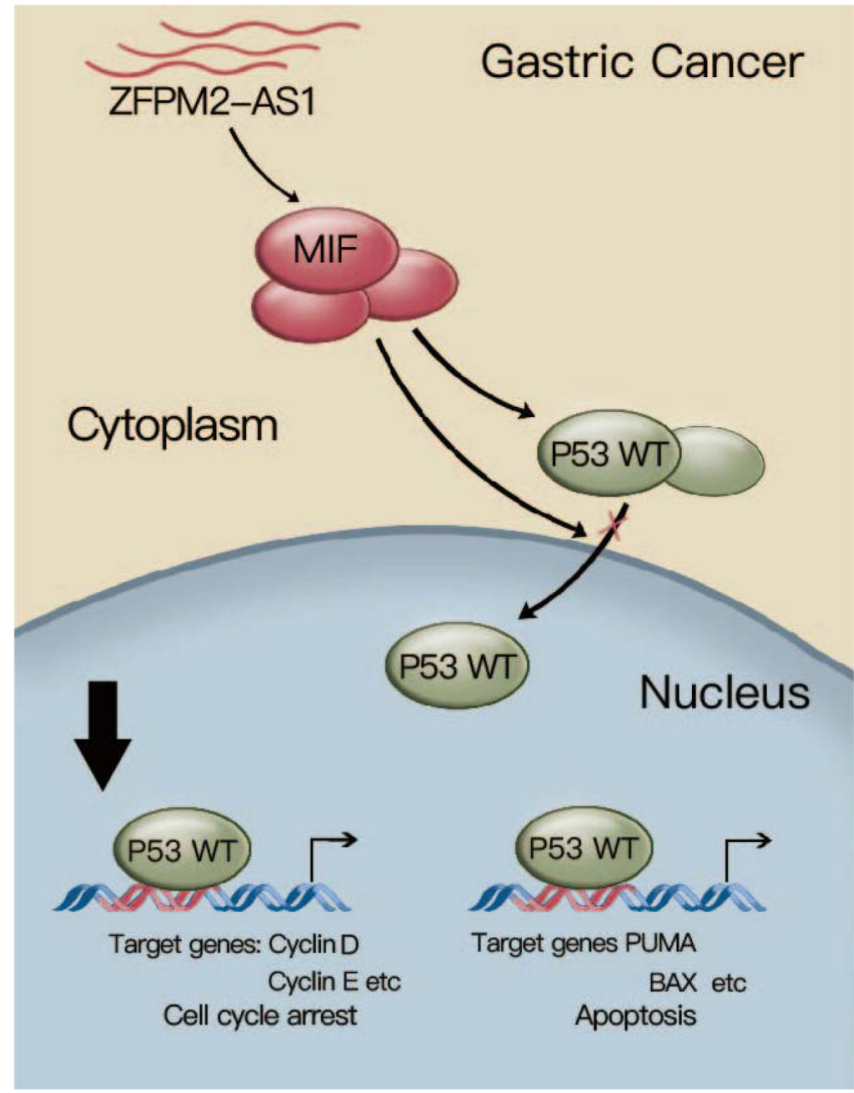

Figure 7. Schematic of gastric cancer progression

Upregulation of ZFPM2-AS1 expression causes increased MIF expression, which in turn suppresses the activation and nuclear translocation of p53 protein. Dysregulation of the p53 signaling pathway increases the proliferation and survival of gastric cancer cells. 